OPEN ACCESS

Edited by: Alessandro Russo,

University of Magna Graecia, Italy

Reviewed by:

Daniele Roberto Giacobbe, Azienda Ospedaliera Universitaria San Martino (IRCCS), Italy Alice Picciarella,

Policlinico Casilino, Italy

*Correspondence:

Guiyi Liao

liaoguiyi@ahmu.edu.cn

${ }^{\dagger}$ These authors have contributed equally to this work

Specialty section: This article was submitted to Clinical Microbiology, a section of the journal Frontiers in Cellular and Infection Microbiology

Received: 16 June 2021 Accepted: 24 September 2021 Published: 08 October 2021

Citation:

Zhang F, Zhong J, Ding H, Pan J, Yang J, Lan T, Chen Y and Liao G (2021) Analysis of Risk Factors for Carbapenem-Resistant Klebsiella pneumoniae Infection and Its Effect on the Outcome of Early Infection After Kidney Transplantation. Front. Cell. Infect. Microbiol. 11:726282. doi: 10.3389/fcimb.2021.726282

\section{Analysis of Risk Factors for Carbapenem-Resistant Klebsiella pneumoniae Infection and Its Effect on the Outcome of Early Infection After Kidney Transplantation}

\author{
Fei Zhang ${ }^{1,2,3 \dagger}$, Jinbiao Zhong ${ }^{1,2,3 \dagger}$, Handong Ding ${ }^{1,2,3 \dagger}$, Jiashan Pan ${ }^{1,2,3}$, Jing Yang ${ }^{1,2,3}$, \\ Tianchi Lan ${ }^{1,2,3}$, Yiding Chen ${ }^{1,2,3}$ and Guiyi Liao ${ }^{1,2,3^{*}}$ \\ ${ }^{1}$ Department of Urology, The First Affiliated Hospital of Anhui Medical University, HeFei, China, ${ }^{2}$ Institute of Urology, \\ The First Affiliated Hospital of Anhui Medical University, HeFei, China, ${ }^{3}$ Anhui Province Key Laboratory of Genitourinary \\ Diseases, Anhui Medical University, HeFei, China
}

Background: Infections remain a major cause of morbidity and mortality in kidney transplant (KT) recipients. This study was performed to identify the overall prevalence of early infections, prevalence of carbapenem-resistant Klebsiella pneumoniae (CRKP) infection after $\mathrm{KT}$, one-year postoperative mortality in patients with early infections and risk factors for CRKP infections.

Methods: We conducted a retrospective study of all patients who received KT in our hospital between January 2017 and December 2019. We evaluated the demographic, clinical, infection characteristics and the one-year postoperative outcomes.

Results: Among the 419 patients who received KT between January 2017 and December 2019, 150 patients had at least one infection within 90 days after KT. The total prevalence of early infections was $36.1 \%$ (150/415), the prevalence of early CRKP infections was $10.4 \%$ (43/415), and the one-year postoperative mortality was 15.3\% (23/ $150)$ in patients with early infections. The risk factors independently related to one-year postoperative mortality were mechanical ventilation $(\mathrm{MV})>48 \mathrm{~h}$ (Odds ratio $(\mathrm{OR})=13.879$, 95\%Confidence interval (Cl): 2.265 85.035; $\mathrm{P}=0.004$ ) and CRKP infection (OR=6.751, 95\% Cl: 1.051 43.369; $\mathrm{P}=0.044) . \mathrm{MV}>48 \mathrm{~h}$ was independently related to CRKP infection (OR=3.719, 95\% Cl: 1.024 13.504; $\mathrm{P}=0.046)$. Kaplan-Meier survival curves showed that the one-year survival rate of patients infected with CRKP in the early postoperative stage was significantly lower than that of uninfected patients.

Conclusions: In general, the prevalence of early infections after KT is high, and CRKP infection is closely correlated with poor prognosis. The effective prevention and treatment of CRKP infection is an important way to improve the one-year survival rate after KT.

Keywords: kidney transplant, carbapenem-resistant Klebsiella pneumoniae, early infections, risk factors, multidrug resistance 


\section{INTRODUCTION}

Solid organ transplant (SOT) recipients have a higher risk of infection than other populations, mainly due to the following reasons: transplantation from marginal donors, especially infected donors; surgical intervention; and the use of immunosuppressants (Linares et al., 2010). Infection is a major cause of the high morbidity and mortality observed in KT recipients (Kim, 2014). Bacterial infection is the most common type of infection after transplantation, followed by fungal, viral and protozoal infections (Blair and Kusne, 2005). The vast majority of bacterial infections, most of which are caused by microorganisms in hospitals, occur in the first three months after transplantation (Fishman, 2011).

In recent years, the rates of infection with gram-negative bacteria and multidrug-resistant gram-negative bacteria (MDRGNB) after SOT have increased. It is estimated that $10 \%-20 \%$ of SOT recipients are infected with MDR-GNB (Zhong et al., 2012; Bodro et al., 2013; Ye et al., 2014) of which carbapenem-resistant Klebsiella pneumoniae (CRKP) is the most lethal. In CRKPendemic areas, the mortality rate of SOT recipients infected with CRKP was estimated to be close to $40 \%$, which is approximately 3-5 times higher than that of non-CRKP-infected recipients (Kalpoe et al., 2012; Clancy et al., 2013; Pouch et al., 2015). These data indicate that early infection after transplantation, especially with CRKP, is related to a poor prognosis of the recipient.

This study was performed to identify the overall prevalence of early infections after KT and the risk factors for one-year postoperative mortality in patients with early infections. We also analyzed the prevalence of early postoperative CRKP infection and its risk factors.

\section{MATERIALS AND METHODS}

\section{Study Design and Patient Sample}

This was a retrospective observational study conducted between January 2017 and December 2019, at the First Affiliated Hospital of Anhui Medical University, a tertiary teaching hospital with 2800 beds located in Anhui Province. The primary endpoint of our study was 1-year mortality of patients with early infections post KT, the secondary endpoint was prevalence of CRKP infection within 3 months post KT. The patient consisted of subjects who received KT in our hospital and survived more than 48 hours after transplantation, Patients infected with bacterial and fungal within three months after KT were included in our study. Among these infected patients, they were further divided into two subgroups, patients with early CRKP infections and patients without CRKP infections. Three recipients were excluded

\footnotetext{
Abbreviations: BSIs, bloodstream infections; CMV, cytomegalovirus; CRKP, carbapenem-resistant Klebsiella pneumoniae; DGF, delayed graft function; ICU, intensive care unit; KT, kidney transplant; MV, mechanical ventilation; MDR, multidrug-resistant; MDR-GNB, multidrug-resistant gram-negative bacteria; SOT, Solid organ transplant; SSIs, surgical site infections; UTIs, urinary tract infections.
}

due to loss of follow-up data and one died within 48 hours, finally a total of 415 recipients were included. All recipients were regularly followed up in the outpatient department after transplantation. All the selected recipients received triple immunosuppression (tacrolimus or cyclosporin A, prednisone, and mycophenolate mofetil), and some received additional antithymocyte immunoglobulin. A third-generation cephalosporin/ $\beta$-lactamase inhibitor or semisynthetic penicillin/ $\beta$-lactamase inhibitor was used 1 hour before transplantation, and the same drug was used for at least 7 days after transplantation. This study was approved by our institutional Ethics Review Committee and was conducted in accordance with the principles of the Declaration of Helsinki.

\section{Clinical Data Collection}

The patients' clinical data, including demographics, infection characteristics, and preoperative, intraoperative, and postoperative variables, were reviewed by electronic medical records. The total prevalence of postoperative infections, the prevalence of CRKP infections since the day of transplantation and the one-year postoperative mortality rate were calculated. In the analysis of the risk factors for one-year mortality in the patients with early infections, we evaluated age, sex, donor type, diabetes mellitus, etiology of renal failure, acute rejection, site of infection, delayed graft function (DGF), anti-thymocyte globulin induction, mechanical ventilation $(\mathrm{MV})>48 \mathrm{~h}$, multiple infections, fungal infection, cytomegalovirus (CMV) infection, multifocal infections, mixed infections, multidrug-resistant (MDR) bacterial infection, CRKP infection, postoperative leukopenia, reintervention and the length of ICU(intensive care unit) stay.

For the patients with early CRKP infections, only the first CRKP infection after transplantation were included in the study. We collected patient age, sex, diabetes mellitus, etiology of renal failure, donor type, acute rejection, CMV infection, DGF, antithymocyte globulin induction, postoperative leukopenia, reintervention, the length of ICU stay and MV> $48 \mathrm{~h}$ and assessed them as potential risk factors for CRKP infection.

\section{Definition}

Early infection was defined as an infection occurring in the first three months after transplantation. The standards used to define and classify infections in our study were those proposed by the Centers for Disease Control and Prevention (Horan et al., 2008). In particular, factors such as pneumonia (including ventilatorassociated infections), surgical site infections (SSIs), bloodstream infections (BSIs) (including catheter-associated infections) and urinary tract infections (UTIs) were taken into account. On the basis of clinical suspicion, the culture of bacteria or fungi from blood, sputum (or other respiratory secretions), urine or ascites samples was carried out for diagnostic purposes, and the occurrence of infection was defined based on the combination of a positive culture with clinical manifestations. CRKP was defined as insensitivity to at least one of the carbapenems, with a minimum inhibitory concentration $\geq 2 \mathrm{mg} / \mathrm{mL}$ for ertapenem and $\geq 4 \mathrm{mg} / \mathrm{mL}$ for imipenem or meropenem (Clinical and Laboratory Standards Institute, 2017). Reintervention was 
defined as any postoperative invasive procedure that required local or general anesthesia. DGF was defined as a decrease in daily serum creatinine less than $10 \%$ from the previous day for 3 consecutive days in the first postoperative week or serum creatinine failing to decrease to $400 \mu \mathrm{mol} / \mathrm{L}$ in the first postoperative week (Paloyo et al., 2016). Leukopenia was defined as a leukocyte count less than 3000 cells/ $\mu \mathrm{L}$ detected at least once on a white blood cell test (Hamel et al., 2019).

\section{Microbiology}

When any infection was suspected during the follow-up period, clinical samples were collected for culture, and microbial culture and identification were carried out in accordance with standard bacteriological procedures. Bacteria were cultured and identified with the VITEK-2 system (Biomerieux, Marcy-1 'Etoile, France). The minimum inhibitory concentration was interpreted according to the breakpoint set by the Clinical and Laboratory Standards Institute (2017).

\section{Statistical Analysis}

The statistical analysis was performed using SPSS software [Version 25.0; SPSS Inc., Chicago, IL, USA]. Categorical variables are described as frequencies and percentages. Continuous variables with normal distributions are described as the means and standard deviations; otherwise, they are described as the medians (IQRs). The Kolmogorov-Smirnov test was conducted to evaluate the normality of variable distribution. An independent-samples t-test was conducted to compare the means of two normally distributed variables. The Mann-Whitney U-test was conducted to evaluate nonnormally distributed variables. The $\mathrm{X} 2$ test was conducted to evaluate the difference between categorical variables. The risk factors for oneyear mortality in patients with early infections after KT were identified by univariate and multivariate logistic regression analyses using odds ratios (ORs) and 95\% confidence intervals (CIs). Univariate and multivariate logistic regression analyses were also carried out to identify the risk factors for CRKP infection. Variables that are significant at the univariate level $(\mathrm{P}<0.05)$ were included in the stepwise binary Logistic regression model to analyze the factors affecting the outcome. The 10 -fold cross validation method is used to verify the robustness of the model. Kaplan-Meier analysis and the log-rank test were conducted to evaluate the difference in the one-year survival curves between patients with and without CRKP infections. $\mathrm{P}<0.05$ was considered statistically significant.

\section{RESULTS}

According to the medical records, 419 patients received KT between January 2017 and December 2019, a total of 419 patients received kidney transplantation, three recipients with lost follow-up data and one recipient who died within 48 hours after KT were excluded, 150 of the 415 included recipients recorded at least one infection within 90 days after transplantation. The total prevalence of early infections was $36.1 \%$, and the prevalence of MDR bacterial infections was $22.9 \%$. A total of $69.3 \%$ of the 150 patients received kidneys donated by deceased donors. The most common etiology of renal failure was glomerulonephritis.

\section{Infection Site and Microbial Etiology}

Of the 150 patients, 95 patients $(63.3 \%)$ had infections at only one site, and 55 patients (36.7\%) had infections at $\geq 2$ sites. Of the 226 recorded infections, $67(29.7 \%)$ were pneumonia, 62 (27.4\%) were UTIs, 56 (24.8\%) were SSIs, and 41 (18.1\%) were BSIs. A total of 347 pathogens were isolated from the sites of infection, of which $69.5 \%$ were gram-negative bacteria, $16.4 \%$ were gram-positive bacteria and $14.1 \%$ were fungi. K. pneumoniae $(42.7 \%)$ was the most common pathogen among the gram-negative bacteria, followed by Escherichia coli (10.4\%) and Stenotrophomonas maltophilia (8.7\%). Among the grampositive bacteria, Enterococcus faecium (28\%) was the most common, followed by Staphylococcus epidermidis (19.3\%) and Staphylococcus aureus (14.0\%). Among the fungi, C. glabrata (38.8\%) was the most common. The distribution of bacteria at different infection sites is shown in Figure 1.

In terms of drug resistance, a total of 171 strains of MDR bacteria were isolated from 95 people, and MDR strains accounted for $49.3 \%$ of all isolates. The MDR-GNB bacteria were mainly K. pneumoniae $(57.1 \%)$, E. coli $(11.0 \%)$, and Acinetobacter baumannii (8.4\%). Of the strains of $\mathrm{K}$. pneumoniae, CRKP accounted for $75.7 \%$ and ESBL-producing bacteria accounted for $9.7 \%$. MDR strains accounted for $68.4 \%$ of the isolates of A. baumannii and $60.7 \%$ of those of E. coli. E. faecium (71.4\%) was the main MDR gram-positive bacteria, and MDR strains accounted for $58.8 \%$ of the isolates of E. faecium.

\section{Analysis of Mortality and the Factors Related to the Mortality of the Recipients After KT}

Of the 150 patients with early infections, 23 died within one year after transplantation. A total of $69.6 \%$ died of infectious complications, $17.4 \%$ died of vascular complications, $8.7 \%$ died of neurological complications and $4.3 \%$ died of other types of complications. Approximately $15.3 \%$ of the patients with early postoperative infections died. The overall mean mortality rate after transplantation was 5.5\%. Univariate logistic regression analysis showed that the potential risk factors for mortality within 1 year after transplantation were deceased donors, pneumonia, BSI, DGF, MV $>48 \mathrm{~h}$, multifocal infection, MDR bacterial infection, CRKP infection, leukopenia after transplantation, reintervention after transplantation and the length of ICU stay. Multivariate logistic regression analysis showed that $\mathrm{MV}>48 \mathrm{~h}(\mathrm{OR}=13.879$, 95\% CI: 2.265 85.035; $\mathrm{P}=0.004)$ and CRKP infection $(\mathrm{OR}=6.751$, 95\% CI: 1.051 43.369; $\mathrm{P}=0.044)$ were independent risk factors for mortality within 1 year after surgery, as shown in Table 1.

\section{Analysis of Risk Factors for CRKP Infection After KT}

Of the $415 \mathrm{KT}$ recipients, 43 patients, accounting for $10.4 \%$ of the total population, were infected with CRKP, and the rate of CRKP infection-related mortality was $37.2 \%(16 / 43)$. Of the surviving recipients, 6 underwent graft nephrectomy due to CRKP infection. The median time from transplantation to the first 
A

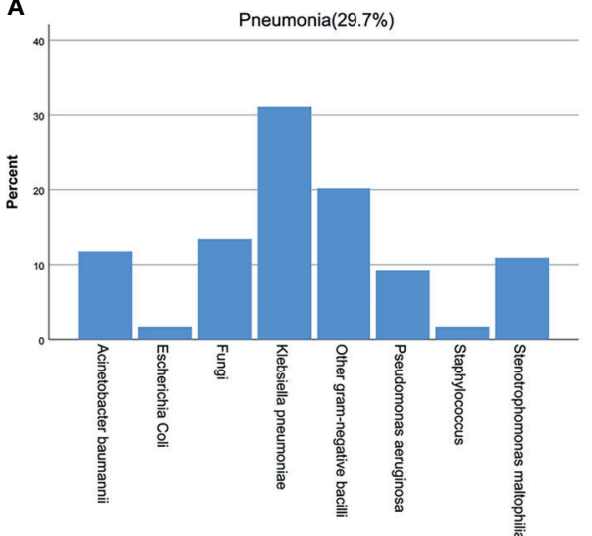

C

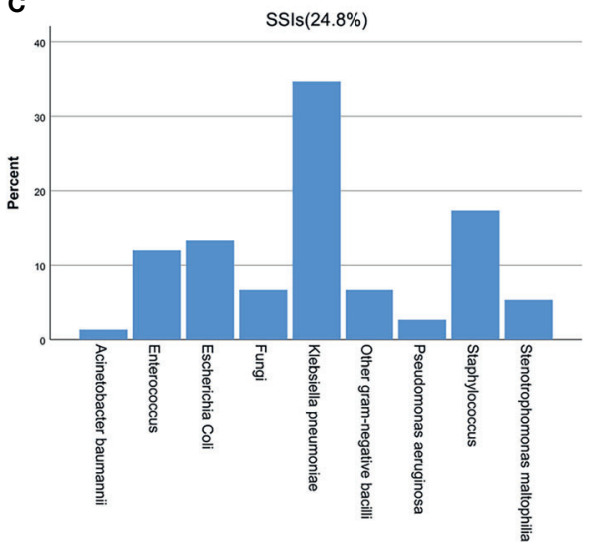

B

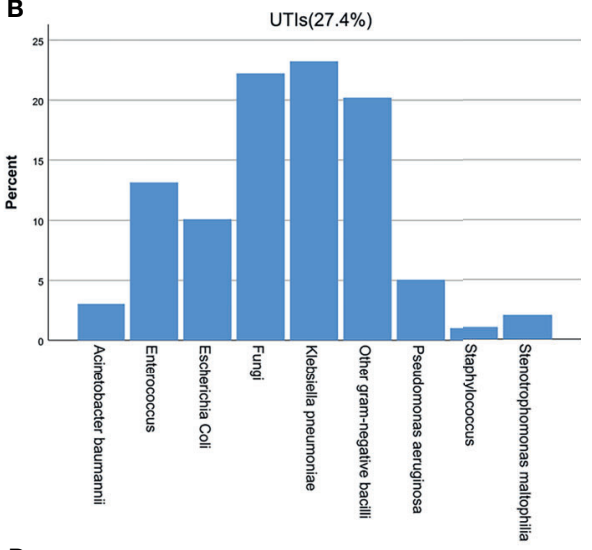

D

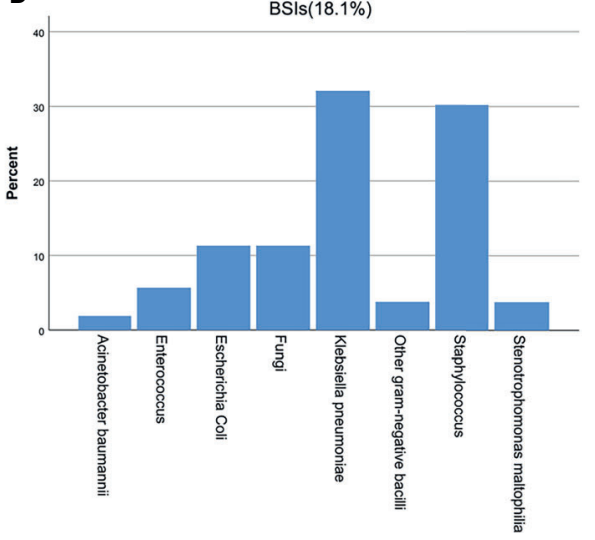

FIGURE 1 | Pathogen distribution at different infected sites after KT. (A) pneumonia, (B) UTIs, (C) SSIs, (D) BSIs.

CRKP infection was 28 days (IQR 17-40 days), In terms of CRKP infection sites, there were 25 cases of pneumonia, 23 cases of SSIs, 15 cases of BSIs and 13 cases of UTIs. 32 (74.4\%) of the 43 patients with CRKP infection received tigecycline based antibiotic regimen, $8(18.6 \%)$ received aminoglycosides based antibiotic regimen, and $3(7.0 \%)$ received ceftazidime- avibatan or Polymyxin antibiotic regimen. Univariate logistic regression analysis showed that the potential risk factors for CRKP infection were DGF, leukopenia after KT, reintervention after transplantation, $\mathrm{MV}>48 \mathrm{~h}$ and the length of ICU stay. Multivariate logistic regression analysis showed that $\mathrm{MV}>48 \mathrm{~h}$ $(\mathrm{OR}=3.719,95 \%$ CI: 1.024-13.504; $\mathrm{P}=0.046)$ was an independent risk factor for CRKP infection, as shown in Table 2. The antibiotic sensitivity of CRKP isolates are shown in Table 3. According to Kaplan-Meier analysis, the overall survival rate of CRKP-infected patients was significantly lower than that of nonCRKP-infected patients, as shown in Figure 2. $(\mathrm{P}<0.001)$.

\section{DISCUSSION}

Due to their poor physical function, frequent need for antibacterial treatment, multiple hospitalizations, and immunosuppression, SOT recipients have a higher risk of infection with MDR bacteria (Barchiesi et al., 2016; Pouch and Satlin, 2017). In our study, we investigated the prevalence of early bacterial and fungal infections and the distribution of pathogens after KT. Of the 415 recipients, 150 (36.1\%) had at least one infection recorded within 90 days after transplantation, of whom 23 died within a year after transplantation. The overall infection rate was $36.1 \%$. To date, no study has reported the overall prevalence of early infections after KT. The overall postoperative infection rate in our study was higher than that after liver transplantation, which Barchiesi et al (Barchiesi et al., 2016) reported was $26.7 \%$, possibly due to the inclusion of donor-derived infections in our study. Although we investigated 20 demographic, clinical and microbiological characteristics, only two factors were independently associated with 1-year mortality, namely, MV $>48 \mathrm{~h}$ and CRKP infection. A possible explanation for this finding is that patients requiring $\mathrm{MV}$ are more likely to use invasive devices and be admitted to intensive care units, both of which increase the risk of CRKP infection (Patel et al., 2008; Giannella et al., 2015). Pneumonia and BSIs were identified in univariate analysis, but they did not remain significant in multivariate analysis, indicating that the type of infection had no effect on the results.

In our study, CRKP infection was found to be an independent risk factor for one-year mortality after KT. The same conclusion 
TABLE 1 | Univariate and multivariate analysis of risk factors for one-year mortality in 150 kidney transplant recipients with early infection.

\begin{tabular}{|c|c|c|c|c|c|c|}
\hline \multirow[t]{2}{*}{ Variable } & \multicolumn{2}{|c|}{ Kidney transplant } & \multicolumn{2}{|c|}{ Univariate analysis } & \multicolumn{2}{|c|}{ Multivariate analysis } \\
\hline & Death $(\mathrm{N}=23)$ & Survival $(\mathrm{N}=127)$ & OR $(95 \% \mathrm{Cl})$ & $P$ value & OR $(95 \% \mathrm{Cl})$ & $\mathbf{P}$ value \\
\hline Mean age (years) & $41.39 \pm 8.93$ & $39.02 \pm 9.93$ & $1.025(0.979-1.074)$ & 0.285 & & \\
\hline Male, Sex, n (\%) & 15 (65.22) & 93 (73.23) & $1.459(0.568-3.748)$ & 0.433 & & \\
\hline Deceased donors, n (\%) & $21(91.30)$ & $83(65.35)$ & $5.566(1.247-24.840)$ & 0.024 & $1.551(0.223-10.777)$ & 0.657 \\
\hline Diabetes mellitus, n (\%) & $8(34.78)$ & $30(23.62)$ & $1.724(0.666-4.462)$ & 0.261 & & \\
\hline \multicolumn{7}{|l|}{ Etiology of renal failure, $\mathrm{n}(\%)$} \\
\hline DM & $5(21.74)$ & $15(11.81)$ & $2.074(0.671-6.407)$ & 0.205 & & \\
\hline HTA & $5(21.74)$ & $30(23.62)$ & $0.898(0.307-2.624)$ & 0.844 & & \\
\hline GD & $7(30.43)$ & $40(31.50)$ & $0.952(0.363-2.495)$ & 0.920 & & \\
\hline Others & $6(26.09)$ & $42(33.07)$ & $0.714(0.262-1.944)$ & 0.510 & & \\
\hline Acute rejection, n (\%) & $11(47.83)$ & $58(45.67)$ & $1.091(0.448-2.654)$ & 0.849 & & \\
\hline \multicolumn{7}{|l|}{ Types of infection, $n(\%)$} \\
\hline Pneumonia & 19 (82.61) & $48(37.80)$ & 7.818 (2.509-24.354) & $<0.001$ & $2.086(0.343-12.678)$ & 0.425 \\
\hline SSIs & $9(39.13)$ & $47(37.00)$ & $1.094(0.440-2.723)$ & 0.846 & & \\
\hline BSls & $11(47.83)$ & $30(23.62)$ & 2.964 (1.187-7.399) & 0.020 & 3.867 (0.632-23.655) & 0.143 \\
\hline UTIS & $11(47.83)$ & $51(40.16)$ & $1.366(0.560-3.332)$ & 0.493 & & \\
\hline DGF, n (\%) & $14(60.87)$ & $33(25.98)$ & 4.431 (1.754-11.192) & 0.002 & $1.962(0.408-9.421)$ & 0.400 \\
\hline ATG induction, n (\%) & $7(30.43)$ & 25 (16.69) & 1.785 (0.663-4.804) & 0.251 & & \\
\hline $\mathrm{MV}>48 \mathrm{~h}, \mathrm{n} \quad(\%)$ & 18 (78.26) & $10(7.87)$ & 42.120 (12.908-137.439) & $<0.001$ & 13.879 (2.265-85.035) & 0.004 \\
\hline Multiple infections ${ }^{a}, \mathrm{n}(\%)$ & $6(26.09)$ & $30(23.62)$ & $1.141(0.413-3.155)$ & 0.799 & & \\
\hline Fungal infections, n (\%) & $6(26.09)$ & $29(22.83)$ & 1.193 (0.431-3.303) & 0.735 & & \\
\hline CMV, n (\%) & $6(26.09)$ & $35(27.56)$ & $0.928(0.338-2.544)$ & 0.884 & & \\
\hline Multifocal infections ${ }^{\mathrm{b}}, \mathrm{n}(\%)$ & 19 (82.61) & 36 (28.35) & 12.007 (3.820-37.738) & $<0.001$ & $1.182(0.195-7.169)$ & 0.856 \\
\hline Mixed infectionsc ${ }^{c}, \mathrm{n}(\%)$ & $5(21.74)$ & $23(18.11)$ & $1.256(0.423-3.732)$ & 0.683 & & \\
\hline Multidrug resistant bacteria infections, $\mathrm{n}(\%)$ & $20(86.96)$ & 75 (59.06) & $4.622(1.306-16.360)$ & 0.018 & $0.232(0.022-2.395)$ & 0.220 \\
\hline CRKP infection, $n(\%)$ & $16(69.57)$ & $27(21.26)$ & $8.466(3.162-22.662)$ & $<0.001$ & $6.751(1.051-43.369)$ & 0.044 \\
\hline Leukopenia after KT, n (\%) & $11(47.83)$ & $8(6.30)$ & $13.635(4.598-40.438)$ & $<0.001$ & $1.238(0.203-7.548)$ & 0.817 \\
\hline Postoperative reintervention, n (\%) & $18(78.26)$ & $34(26.77)$ & $9.847(3.392-28.588)$ & $<0.001$ & $2.817(0.591-13.429)$ & 0.194 \\
\hline Length of ICU stay (days) & $16.96 \pm 19.95$ & $3.18 \pm 7.61$ & $1.114(1.051-1.182)$ & $<0.001$ & $0.995(0.951-1.041)$ & 0.827 \\
\hline
\end{tabular}

TABLE 2 | Univariate and multivariate analysis of the risk of carbapenem-resistant Klebsiella pneumoniae infection in 150 kidney transplant recipients.

\begin{tabular}{|c|c|c|c|c|c|c|}
\hline \multirow[b]{2}{*}{ Variable } & \multicolumn{2}{|c|}{ CRKP infection } & \multicolumn{2}{|c|}{ Univariate analysis } & \multicolumn{2}{|c|}{ Multivariate analysis } \\
\hline & Yes $(\mathrm{N}=43)$ & No $(\mathrm{N}=107)$ & OR $(95 \% \mathrm{Cl})$ & $P$ value & OR $(95 \% \mathrm{Cl})$ & $P$ value \\
\hline Mean age (years) & $42.30 \pm 7.92$ & $38.21 \pm 10.25$ & $1.045(1.006-1.086)$ & 0.022 & $1.030(0.986-1.076)$ & 0.181 \\
\hline Male, Sex, n (\%) & $32(74.42)$ & $76(71.03)$ & $0.843(0.378-1.880)$ & 0.676 & & \\
\hline Deceased donors, n (\%) & $35(81.40)$ & 69 (64.49) & 2.409 (1.015-5.717) & 0.046 & $1.206(0.442-3.292)$ & 0.715 \\
\hline Diabetes mellitus, $n(\%)$ & $13(30.23)$ & $25(23.36)$ & $1.421(0.645-3.132)$ & 0.383 & & \\
\hline \multicolumn{7}{|l|}{ Etiology of renal failure, $\mathrm{n}(\%)$} \\
\hline DM & $7(16.28)$ & $13(12.15)$ & $1.406(0.519-3.806)$ & 0.502 & & \\
\hline HTA & $9(20.93)$ & $26(24.30)$ & $0.825(0.350-1.944)$ & 0.659 & & \\
\hline GD & $14(32.56)$ & $33(30.84)$ & $1.083(0.507-2.311)$ & 0.838 & & \\
\hline Others & $13(30.23)$ & $35(32.71)$ & $0.891(0.414-1.917)$ & 0.769 & & \\
\hline Acute rejection, n (\%) & $22(51.16)$ & 47 (43.93) & $1.337(0.658-2.719)$ & 0.422 & & \\
\hline DGF, n (\%) & 19 (44.19) & $28(26.17)$ & $2.234(1.065-4.683)$ & 0.033 & $1.196(0.468-3.060)$ & 0.708 \\
\hline ATG induction, n (\%) & $7(16.28)$ & $25(23.36)$ & $0.638(0.253-1.609)$ & 0.341 & & \\
\hline$M V>48 h, n(\%)$ & $18(41.86)$ & $10(9.34)$ & $6.984(2.870-16.995)$ & $<0.001$ & 3.719 (1.024-13.504) & 0.046 \\
\hline CMV, n (\%) & $8(18.60)$ & $33(30.84)$ & $0.513(0.215-1.224)$ & 0.132 & & \\
\hline Leukopenia after KT, n (\%) & $13(30.23)$ & $6(5.61)$ & 7.294 (2.553-20.838) & $<0.001$ & $2.721(0.737-10.050)$ & 0.133 \\
\hline Postoperative reintervention, $\mathrm{n}(\%)$ & 21 (48.84) & $31(28.97)$ & $2.340(1.128-4.853)$ & 0.022 & $0.903(0.334-2.437)$ & 0.840 \\
\hline Length of ICU stay (days) & $10.19 \pm 19.34$ & $3.33 \pm 4.94$ & $1.064(1.015-1.114)$ & 0.009 & $1.005(0.961-1.052)$ & 0.825 \\
\hline
\end{tabular}

DM, diabetes mellitus; HTA, hypertension; GD, glomerular disease; DGF, delayed graft function; ATG, anti-thymocyte globulin; MV, mechanical ventilation.

Bold values indicated that these variables were significant in univariate and multivariate analysis $(P<0.05)$.

was reached in two recent studies on liver transplantation. Those two studies showed that when liver transplant recipients were infected with CRKP, the 1-year survival rate decreased significantly from $86 \%$ to $29 \%$ and from $93 \%$ to $55 \%$ (Kalpoe et al., 2012; Pereira et al., 2015). In this study, a total of 43 recipients had CRKP infections, yielding an infection rate of 
TABLE 3 | Antimicrobial susceptibility of isolates from patients with carbapenem-resistant klebsiella pneumoniae infection.

\begin{tabular}{lcc}
\hline Antibiotic & Number of isolates tested (N) & Susceptible (\%) \\
\hline Ceftazidime & 43 & $0.0 \%$ \\
Levofloxacin & 43 & $9.3 \%$ \\
Gentamycin & 40 & $12.5 \%$ \\
Aztreonam & 43 & $0.0 \%$ \\
Imipenem & 43 & $0.0 \%$ \\
Meropenem & 43 & $0.0 \%$ \\
Amikacin & 40 & $20.0 \%$ \\
Polymyxin & 42 & $97.6 \%$ \\
Tigecycline & 43 & $100.0 \%$
\end{tabular}

$10.4 \%$. In terms of the prevalence, published studies have reported different CRKP infection rates ranging from 3\% to 11.2\% (Clancy et al., 2013; Varotti et al., 2017; Bias et al., 2018). In our study, it was found that the prevalence of CRKP infection was very high, possibly because our institute is located in an area with a high incidence of CRKP infection. CHINET data show that the drug resistance rates of $\mathrm{K}$. pneumoniae to imipenem and meropenem increased from $2.9 \%$ to $23.7 \%$ and $3 \%$ to $25 \%$ in China from 2005 to 2019, respectively, and the incidence of CRKP infection has also increased sharply worldwide in the past decade (, 2018)

Of the 43 patients with CRKP infections, 16 (37.2\%) died. Previous studies showed that the overall mortality of transplant recipients with CRKP infections was as high as 40\% 75\% (Mazza et al., 2017; Bias et al., 2018; Wang et al., 2020). In our study, the Kaplan-Meier curves showed that the one-year survival rate after transplantation in the CRKP-infected group was significantly lower than that in the control group. This confirms previous the data reported in in the literature, which generally suggest that CRKP infection has a negative effect on outcomes in renal transplant patients. The resistance of CRKP to most antibiotics is an important factor affecting the high mortality rate. In addition, the toxicity and side effects of antibiotics, immunocompromise, graft insufficiency requiring hemodialysis and mixed infections are also important reasons for the high mortality rate. Some studies have reported that allograft nephrectomy can improve the antimicrobial treatment success rate (Clancy et al., 2013; Simkins et al., 2014). In our study, 6 of the surviving patients underwent graft nephrectomy, complete debridement and drainage, which shows that drainage, debridement and other mechanical methods of removing infected lesions are important auxiliary means of managing CRKP infections.

Other studies have also predicted risk factors for CRKP infections, such as DGF, diabetes (Varotti et al., 2017; Taminato et al., 2019; Vigara et al., 2020). In our analysis, these factors were not associated with a higher risk of CRKP infection. Instead, $\mathrm{MV}>48 \mathrm{~h}$ was found to be an independent risk factor for CRKP infection, which is consistent with the results obtained in previous studies (Patel et al., 2008; Giannella et al., 2015). The graft loss rate and mortality rate in the control group we selected were higher than the average rates at the transplant center, and their condition was more severe than that of the recipients without any infections. We did not select KT recipients who did not have infections as the control group to facilitate the identification of the factors associated with CRKP infection. In the analysis of the risk factors for CRKP infection, donor-derived infections have been reported as a mechanism of transmission (Mularoni et al., 2015; Varotti et al., 2016).

This study has several limitations. Firstly, the results of this retrospective study, which only included patients from a single institute, may not be applicable to KT recipients in other institutions or at other locations. Secondly, in the analysis of risk factors for CRKP infection, various studies have emphasized the importance of the colonization status of patients before and after transplantation (Giannella et al., 2015; Mazza et al., 2017). Unfortunately, we have not been able to collect these data owing to the absence of routine monitoring and screening. Thirdly, we

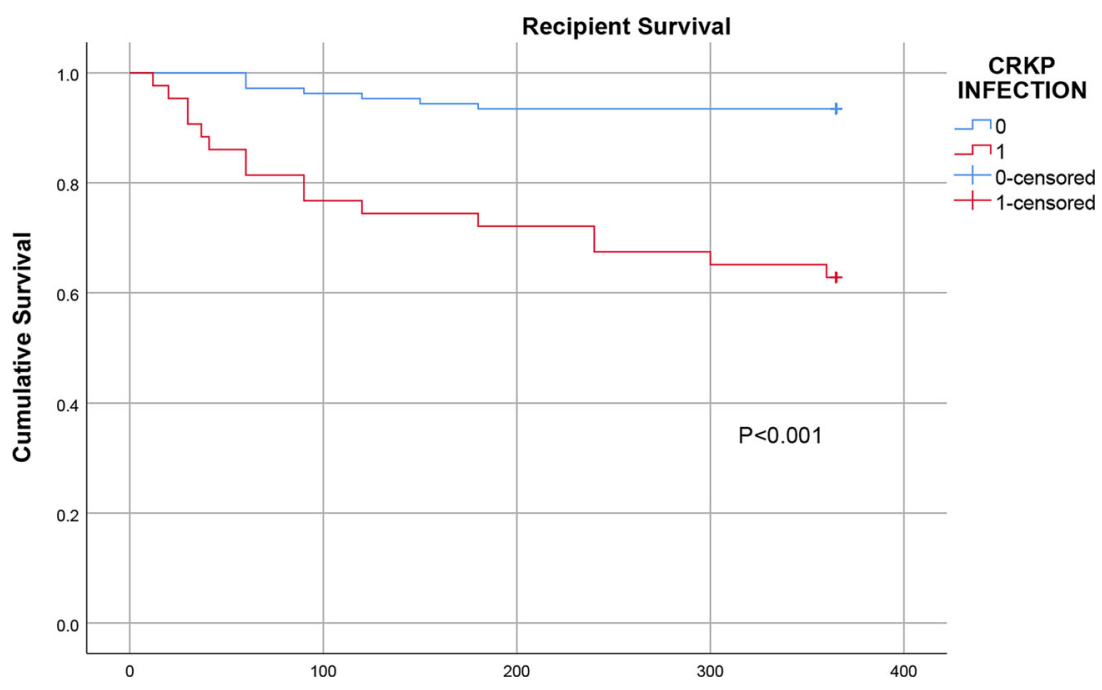

FIGURE 2 | Comparison of the one-year survival rate of infected and uninfected CRKP among early KT recipients. 
did not carry out gene identification and drug resistance mechanism detection of CRKP strain.

\section{CONCLUSION}

In summary, our research showed that the prevalence of CRKP infection is higher in KT recipients. Patients who undergo MV> $48 \mathrm{~h}$ are more likely to develop CRKP infections. CRKP infections negatively affect the prognosis of KT recipients and significantly reduces their survival. Therefore, appropriate isolation and intervention should be performed for high-risk patients to prevent the spread of drug-resistant bacteria.

\section{DATA AVAILABILITY STATEMENT}

The datasets used and/or analysed during the current study are available from the corresponding author on reasonable request.

\section{ETHICS STATEMENT}

The studies involving human participants were reviewed and approved by the ethics committee of The First Affiliated Hospital of Anhui Medical University. Written informed consent for participation was not required for this study in accordance with the national legislation and the institutional

\section{REFERENCES}

(2018). Vital Signs: Carbapenem-Resistant Enterobacteriaceae. Morbidity Mortality Weekly Rep. 63.

Barchiesi, F., Montalti, R., Castelli, P., Nicolini, D., Staffolani, S., Mocchegiani, F., et al. (2016). Carbapenem-Resistant Klebsiella Pneumoniae Influences the Outcome of Early Infections in Liver Transplant Recipients. BMC Infect. Dis. 16 (1), 538. doi: 10.1186/s12879-016-1876-5

Bias, T. E., Malat, G. E., Lee, D. H., Sharma, A., and Doyle, A. M. (2018). Clinical Outcomes Associated With Carbapenem Resistant Klebsiella Pneumoniae (CRKP) in Abdominal Solid Organ Transplant (SOT) Recipients. Infect. Dis. (Lond). Jan 50 (1), 67-70. doi: 10.1080/23744235.2017.1354259

Blair, J. E., and Kusne, S. (2005). Bacterial, Mycobacterial, and Protozoal Infections After Liver Transplantation-Part I. Liver Transpl. 11 (12), 1452-1459. doi: $10.1002 / 1 \mathrm{t} .20624$

Bodro, M., Sabe, N., Tubau, F., Lladó, L., Baliellas, C., Roca, J., et al. (2013). Risk Factors and Outcomes of Bacteremia Caused by Drug-Resistant ESKAPE Pathogens in Solid-Organ Transplant Recipients. Transplantation 96 (9), 843-849. doi: 10.1097/TP.0b013e3182a049fd

Clancy, C. J., Chen, L., Shields, R. K., Zhao, Y., Cheng, S., Chavda, K. D., et al. (2013). Epidemiology and Molecular Characterization of Bacteremia Due to Carbapenem-Resistant Klebsiella Pneumoniae in Transplant Recipients. Am. J. Transplant. 13 (10), 2619-2633. doi: 10.1111/ajt.12424

Clinical and Laboratory Standards Institute (2017). CLSI. Performance Standards for Antimicrobial Susceptibility Testing. Wayne: Twenty-Seventh Informational Supplement. M100- S27.

Fishman, J. A. (2011). Infections in Immunocompromised Hosts and Organ Transplant Recipients: Essentials. Liver Transpl. 17 (Suppl 3), S34-S37. doi: 10.1002/lt.22378

Giannella, M., Bartoletti, M., Morelli, M. C., Tedeschi, S., Cristini, F., Tumietto, F., et al. (2015). Risk Factors for Infection With Carbapenem-Resistant Klebsiella Pneumoniae After Liver Transplantation: The Importance of Pre- and Posttransplant Colonization. Am. J. Transplant. 15 (6), 1708-1715. doi: 10.1111/ajt.13136 requirements. Written informed consent was not obtained from the individual(s) for the publication of any potentially identifiable images or data included in this article.

\section{AUTHOR CONTRIBUTIONS}

FZ, JZ, and HD: study design, statistical analysis, data interpretation, manuscript preparation, literature search. JP: data collection. JY: data collection. TL: data collection. YC: data collection. GL: study design, provision of materials and resources, data interpretation. All authors contributed to the article and approved the submitted version.

\section{FUNDING}

This work received funding from the Natural Science Foundation of Anhui Province (Grant No. 1508085SMH226).

\section{ACKNOWLEDGMENTS}

The authors are grateful to their friend Yuyang Zhang for the assistance provided.

Hamel, S., Kuo, V., Sawinski, D., Johnson, D., Bloom, R. D., Bleicher, M., et al (2019). Single-Center, Real-World Experience With Granulocyte ColonyStimulating Factor for Management of Leukopenia Following Kidney Transplantation. Clin. Transplant. 33 (6), e13541. doi: 10.1111/ctr.13541

Horan, T. C., Andrus, M., and Dudeck, M. A. (2008). CDC/NHSN Surveillance Definition of Health Care-Associated Infection and Criteria for Specific Types of Infections in the Acute Care Setting. Am. J. Infect. Control 36 (5), 309-332. doi: 10.1016/j.ajic.2008.03.002

Kalpoe, J. S., Sonnenberg, E., Factor, S. H., del Rio Martin, J., Schiano, T., Patel, G., et al. (2012). Mortality Associated With Carbapenem-Resistant Klebsiella Pneumoniae Infections in Liver Transplant Recipients. Liver Transpl. 18 (4), 468-474. doi: 10.1002/lt.23374

Kim, S. I. (2014). Bacterial Infection After Liver Transplantation. World J. Gastroenterol. 20 (20), 6211-6220. doi: 10.3748/wjg.v20.i20.6211

Linares, L., Cervera, C., Hoyo, I., Sanclemente, G., Marco, F., Cofán, F., et al. (2010). Klebsiella Pneumoniae Infection in Solid Organ Transplant Recipients: Epidemiology and Antibiotic Resistance. Transplant. Proc. 42 (8), 2941-2943. doi: 10.1016/j.transproceed.2010.07.080

Mazza, E., Prosperi, M., Panzeri, M. F., Limuti, R., Nichelatti, M., and De Gasperi, A. (2017). Carbapenem-Resistant Klebsiella Pneumoniae Infections Early After Liver Transplantation: A Single-Center Experience. Transplant. Proc. 49 (4), 677-681. doi: 10.1016/j.transproceed.2017.02.028

Mularoni, A., Bertani, A., Vizzini, G., Gona, F., Campanella, M., Spada, M., et al. (2015). Outcome of Transplantation Using Organs From Donors Infected or Colonized With Carbapenem-Resistant Gram-Negative Bacteria. Am. J. Transplant. 15 (10), 2674-2682. doi: 10.1111/ajt.13317

Paloyo, S., Sageshima, J., Gaynor, J. J., Chen, L., Ciancio, G., and Burke, G. W. (2016). Negative Impact of Prolonged Cold Storage Time Before Machine Perfusion Preservation in Donation After Circulatory Death Kidney Transplantation. Transpl Int. 29 (10), 1117-1125. doi: 10.1111/tri.12818

Patel, G., Huprikar, S., Factor, S. H., Jenkins, S. G., and Calfee, D. P. (2008). Outcomes of Carbapenem-Resistant Klebsiella Pneumoniae Infection and the Impact of Antimicrobial and Adjunctive Therapies. Infect. Control Hosp Epidemiol. 29 (12), 1099-1106. doi: 10.1086/592412 
Pereira, M. R., Scully, B. F., Pouch, S. M., Uhlemann, A. C., Goudie, S., Emond, J. E., et al. (2015). Risk Factors and Outcomes of Carbapenem-Resistant Klebsiella Pneumoniae Infections in Liver Transplant Recipients. Liver Transpl. 21 (12), 1511-1519. doi: 10.1002/lt.24207

Pouch, S. M., Kubin, C. J., Satlin, M. J., Tsapepas, D. S., Lee, J. R., Dube, G., et al. (2015). Epidemiology and Outcomes of Carbapenem-Resistant Klebsiella Pneumoniae Bacteriuria in Kidney Transplant Recipients. Transpl Infect. Dis. 17 (6), 800-809. doi: 10.1111/tid.12450

Pouch, S. M., and Satlin, M. J. (2017). Carbapenem-Resistant Enterobacteriaceae in Special Populations: Solid Organ Transplant Recipients, Stem Cell Transplant Recipients, and Patients With Hematologic Malignancies. Virulence 8 (4), 391-402. doi: 10.1080/21505594.2016.1213472

Simkins, J., Muggia, V., Cohen, H. W., and Minamoto, G. Y. (2014). CarbapenemResistant Klebsiella Pneumoniae Infections in Kidney Transplant Recipients: A Case-Control Study. Transpl Infect. Dis. 16 (5), 775-782. doi: 10.1111/tid.12276

Taminato, M., Fram, D., Pereira, R. R. F., Sesso, R., Belasco, A., Pignatari, A. C., et al. (2019). Infection Related to Klebsiella Pneumoniae Producing Carbapenemase in Renal Transplant Patients. Rev. Bras. Enferm. 72 (3), 760-766. doi: 10.1590/00347167-2019-0009

Varotti, G., Dodi, F., Marchese, A., Terulla, A., Bertocchi, M., and Fontana, I. (2016). Fatal Donor-Derived Carbapenem-Resistant Klebsiella Pneumoniae Infection in a Combined Kidney-Pancreas Transplantation. Case Rep. Transpl. 2016, 7920951. doi: 10.1155/2016/7920951

Varotti, G., Dodi, F., Terulla, A., Santori, G., Mariottini, G., Bertocchi, M., et al. (2017). Impact of Carbapenem-Resistant Klebsiella Pneumoniae (CR-KP) Infections in Kidney Transplantation. Transpl Infect. Dis. 19 (6), 10.1111/tid.12757. doi: 10.1111/tid.12757

Vigara, L. A., Villanego, F., Cazorla, J. M., Naranjo, J., Mínguez, M., Garcia, A. M., et al. (2020). Characteristics and Evolution of Renal Transplant Recipients Infected by Carbapenemase-Producing Klebsiella Pneumoniae. Transplant. Proc. 52 (2), 519-522. doi: 10.1016/j.transproceed.2019.09.017
Wang, Z., Qian, Y., Bai, H., Yang, J., and Li, X. (2020). Allograft Hemorrhage as a Manifestation of Carbapenem-Resistant Klebsiella Pneumonia Infection in Kidney Transplant Recipients: Case Series. Med. (Baltimore) 99 (13), e18982. doi: 10.1097/MD.0000000000018982

Ye, Q. F., Zhao, J., Wan, Q. Q., Qiao, B. B., and Zhou, J. D. (2014). Frequency and Clinical Outcomes of ESKAPE Bacteremia in Solid Organ Transplantation and the Risk Factors for Mortality. Transpl Infect. Dis. 16 (5), 767-774. doi: $10.1111 /$ tid. 12278

Zhong, L., Men, T. Y., Li, H., Peng, Z. H., Gu, Y., Ding, X., et al. (2012). MultidrugResistant Gram-Negative Bacterial Infections After Liver Transplantation Spectrum and Risk Factors. J. Infect. Mar. 64 (3), 299-310. doi: 10.1016/ j.jinf.2011.12.005

Conflict of Interest: The authors declare that the research was conducted in the absence of any commercial or financial relationships that could be construed as a potential conflict of interest.

Publisher's Note: All claims expressed in this article are solely those of the authors and do not necessarily represent those of their affiliated organizations, or those of the publisher, the editors and the reviewers. Any product that may be evaluated in this article, or claim that may be made by its manufacturer, is not guaranteed or endorsed by the publisher.

Copyright (c) 2021 Zhang, Zhong, Ding, Pan, Yang, Lan, Chen and Liao. This is an open-access article distributed under the terms of the Creative Commons Attribution License (CC BY). The use, distribution or reproduction in other forums is permitted, provided the original author(s) and the copyright owner(s) are credited and that the original publication in this journal is cited, in accordance with accepted academic practice. No use, distribution or reproduction is permitted which does not comply with these terms. 